\title{
Solitary water wave interactions for the Forced Korteweg-de Vries equation
}

\author{
M. V. Flamarion ${ }^{1}$ and R. Ribeiro-Jr²
}

\begin{abstract}
${ }^{1}$ UFRPE/Rural Federal University of Pernambuco, UACSA/Unidade Acadêmica do Cabo de Santo Agostinho, BR 101 Sul, 5225, 54503-900, Ponte dos Carvalhos, Cabo de Santo Agostinho, Pernambuco, Brazil.

${ }^{2}$ UFPR/Federal University of Paraná, Departamento de Matemática, Centro Politécnico, Jardim das Américas, Caixa Postal 19081, Curitiba, PR, 81531-980, Brazil.
\end{abstract}

\begin{abstract}
The aim of this work is to study solitary water wave interactions between two topographic obstacles for the forced Korteweg-de Vries equation (fKdV). Focusing on the details of the interactions, we identify regimes in which solitary wave interactions maintain two well separated crests and regimes where the number of local maxima varies according to the laws $2 \rightarrow 1 \rightarrow$ $2 \rightarrow 1 \rightarrow 2$ or $2 \rightarrow 1 \rightarrow 2$. It shows that the geometric Lax-categorization of Korteweg-de Vries $(\mathrm{KdV})$ two-soliton interactions still holds for the fKdV equation.
\end{abstract}

KEYWORDS: Solitary waves collisions, Korteweg-de Vries equation, Solitons.

\section{Introduction}

The forced Korteweg-de Vries equation (fKdV) has been used as a model to describe atmospheric flows encountering topographic obstacles, flow of water over rocks (Baines [1995]), ship waves and ocean waves generated by storms (when a low pressure region moves on the surface of the ocean Johnson 2012]).

Solitary waves have a wide range of applications, for instance, in water waves, optical fibers, superconductive electronics, elementary-particle physics, quantum physics and more recent applications in biology and cosmology (Joseph 2016]). It is well known that the Korteweg-de Vries equation $(\mathrm{KdV})$ is used to describe the propagation and interaction between solitary waves. Studying numerical solutions of the KdV equation Zabusky \& Kruskal 1965 were the first to observe that solitary waves interact during the collision and return to its initial form. They named this type of waves as solitons. This study raised interest to investigate further details of soliton interactions. Since then many works have been done on this topic. It is hard to give a comprehensive overview of contributions. For the interested reader, we mention a few articles which are seminal in this field.

Lax 1968 classified overtaking collisions of two solitons in three categories according to the number of crests observed during the interaction. More precisely, he proved that the type of the collision can be classified according to the ratio of the initial amplitude of the solitons. The categorization given by Lax was verified experimentally by Weidman \& Maxworthy [1978] and numerically by Mirie \& Su 1982] for a higher order model. More recently, Craig et al. [2006] presented a work in which is given a broad review on solitary wave interactions. They investigated numerically and experimentally solitary wave collisions for the Euler equations. Their numerical simulations show that the collisions of two solitary waves fit into the three geometric categories of the KdV two-soliton solutions defined by Lax. However, the algebraic classification based on the ratio of the initial amplitudes is within a different range of the one considered by Lax.

In this paper we investigate numerically in details the interaction of two solitary wave solutions of the fKdV. More precisely, we analyse the interaction of these two waves between obstacles. We find the three geometric categories described by Lax [1968] for the KdV two-soliton interaction. However, our experiments indicate that an algebraic categorization similar to the one presented by Lax is not possible for the fKdV.

This article is organized as follows. In section 2 we present the mathematical formulation of the non-dimensional fKdV equation. The results are presented in section 3 and the conclusion in section 4 .

\section{The forced Korteweg-de Vries equation}

We consider an inviscid, incompressible, homogeneous fluid on a shallow channel with variable topography in the presence of a constant current. The flow of the fluid can be classified by the Froude number $(F)$, which is defined by the ratio of the upstream velocity and the critical speed of shallow water. When the Froude number is near critical $(F \approx 1)$, and the amplitude of the topography is 
small the weakly nonlinear, weakly dispersive model given by the dimensionless forced Korteweg-de Vries equation

$$
\zeta_{t}+f \zeta_{x}-\frac{3}{2} \zeta \zeta_{x}-\frac{1}{6} \zeta_{x x x}=\frac{1}{2} h_{x}(x)
$$

is used to describe the flow over the obstacle (Pratt [1984]; Wu [1987]; Grimshaw \& Maleewong || 2013]; Milewski [2004]; Flamarion et al. [2019]). Here, $\zeta(x, t)$ is the free-surface displacement over the undisturbed surface and $h(x)$ is the obstacle submerged. The parameter $f$ represents a perturbation of the Froude number, i.e, $F=1+\epsilon f$, where $\epsilon>0$ is a small parameter.

It is important to point out that the equation (1) conserves mass $(M(t))$, with

$$
\frac{d M}{d t}=0, \quad \text { where } \quad M(t)=\int_{-\infty}^{+\infty} \zeta(x, t) d x .
$$

When the bottom is flat $\left(h_{x}=0\right)$ a traveling solitary wave solution for (1) is

$$
\zeta(x, t)=A \operatorname{sech}^{2}(k(x-c t)), \quad A=\frac{4}{3} k^{2}, \quad c=f-\frac{1}{2} A .
$$

Notice that when $f=A / 2$ the solution is stationary.

The fKdV equation (1) is solved numerically using a Fourier pseudospectral method with an integrating factor for the linear part, thus avoiding numerical problems due to the higher-order dispersive term. We consider the computational domain with an uniform grid. All derivatives in $x$ are computed spectrally (Trefethen [2001]). Besides, the time evolution is calculated through the Runge-Kutta fourth-order method (Flamarion et al. [2019]).

\section{Results}

We investigate collisions of two solitary waves between the osbtacles. For this purpose the initial condition of (1) is given by a linear sum of two well-separate solitary waves

$$
\zeta(x, 0)=S_{1} \operatorname{sech}^{2}\left(k_{1}(x-\phi)\right)+S_{2} \operatorname{sech}^{2}\left(k_{2}(x+\psi)\right),
$$

where $S_{1}=4 k_{1}^{2} / 3, S_{2}=4 k_{2}^{2} / 3$, and $\phi, \psi$ are positive constants. Our focus is to categorize the collision of two solitary waves into three types in the same spirit as presented in Lax [1968 for the $\mathrm{KdV}$ equation. Studying overtaking $\left(S_{2}>S_{1}\right)$ collisions Lax has classified the details of two-soliton interactions as follows:

(A) For any time $t$ the solution of the KdV has two well-defined and separate crests, and it happens when $S_{2} / S_{1}<(3+\sqrt{5}) / 2 \approx 2.62$.

(B) During the collision the number of local maxima varies according to $2 \rightarrow 1 \rightarrow 2 \rightarrow 1 \rightarrow 2$, and for this case we have $(3+\sqrt{5}) / 2<S_{2} / S_{1}<3$.

(C) In the interaction the number of local maxima changes as $2 \rightarrow 1 \rightarrow 2$ and it occurs for $S_{2} / S_{1}>3$.

After the collision the main notable feature is that the waves are phase shifted, i.e., their crest are slightly shifted from the trajectories of the incoming centers.

In the following simulations we use the parameters $\beta=20, \epsilon=0.01$ and $f=0.34$. Besides, in order to avoid radiation from the topography we sum a term $r(x)$ to the initial condition, where $r$ is the steady solution of the uniform flow.

We start considering the collision of two well-separeted solitary waves that initially have the same amplitude. Details of the wave profile are given in figure1 (top). Initiallly two solitary waves propagate downstream. When the right wave reaches the obstacle its amplitude increases and the wave reflects back upstream. Then the waves collide mimicking a counterpropagating collision. As the right wave approaches the wave with smaller crest the larger wave begins to shrink and the smaller one begins to grow until the two waves interchange their roles (see figure 1 (bottom-right). Throughout the interaction there are two well-defined and separate crests as shown in figure 1 (bottom-left). This behaviour is similar to case (A) of Lax classification. Figure 2 displays the continuation of figure 1(top). After a series of collisions both waves escape out. We point out that the numerical method conserves mass and the relative error is:

$$
\frac{\max _{0 \leq t \leq 10^{4}}|M(t)-M(0)|}{|M(0)|}=\mathcal{O}\left(10^{-16}\right) .
$$

Figure 3 (top) displays the collision of two well-separate solitary waves that initially have different amplitudes. Differently from the previous case there is a period of time in the interaction that 

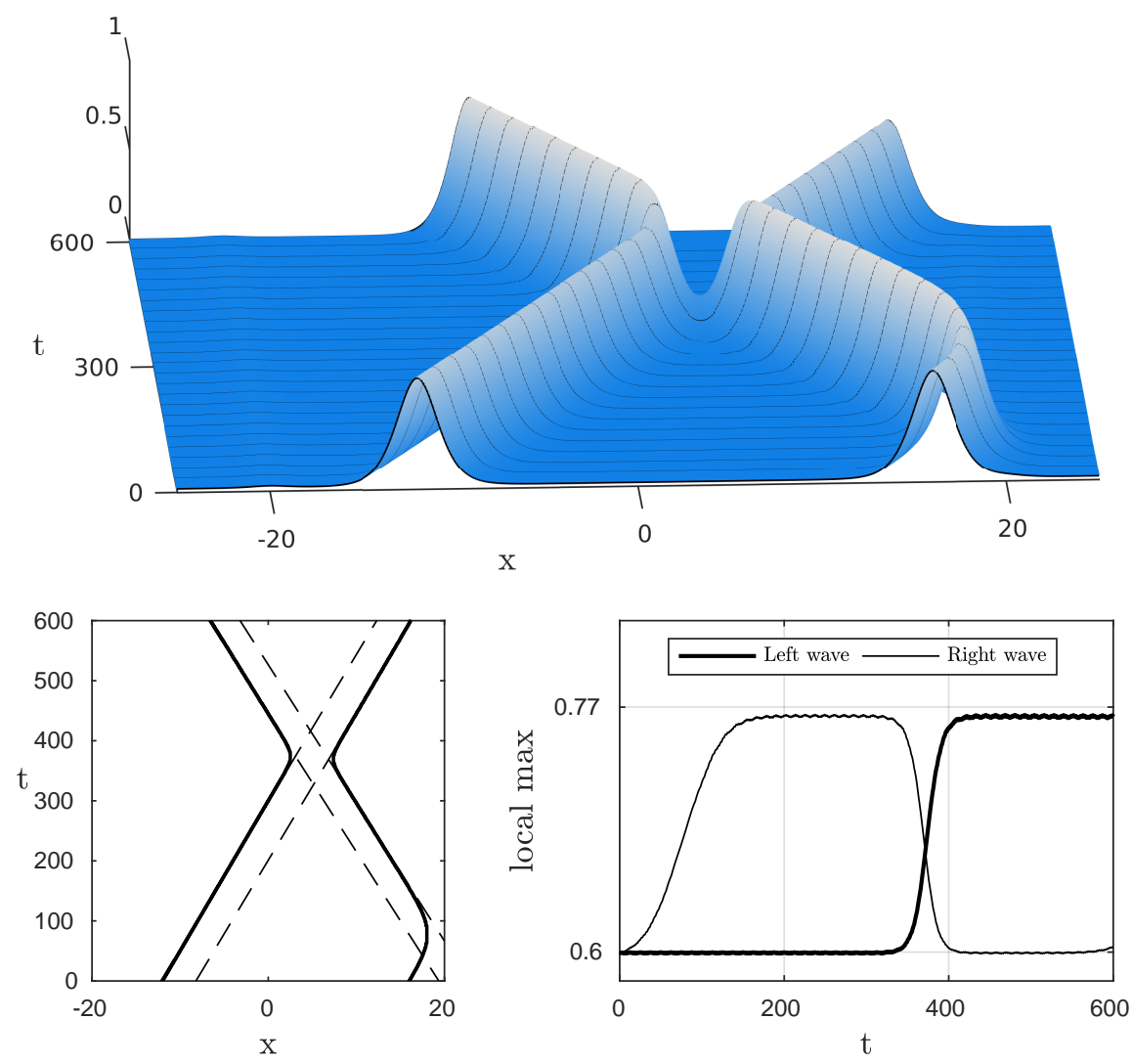

Figure 1: Top: Collision of two well-separate solitary waves - category (A). Bottom (left): Crest trajectories. Bottom (right): The local maxima of the solution as a function of time. Parameters $S_{1}=S_{2}=0.6, \phi=16$ and $\psi=12$.

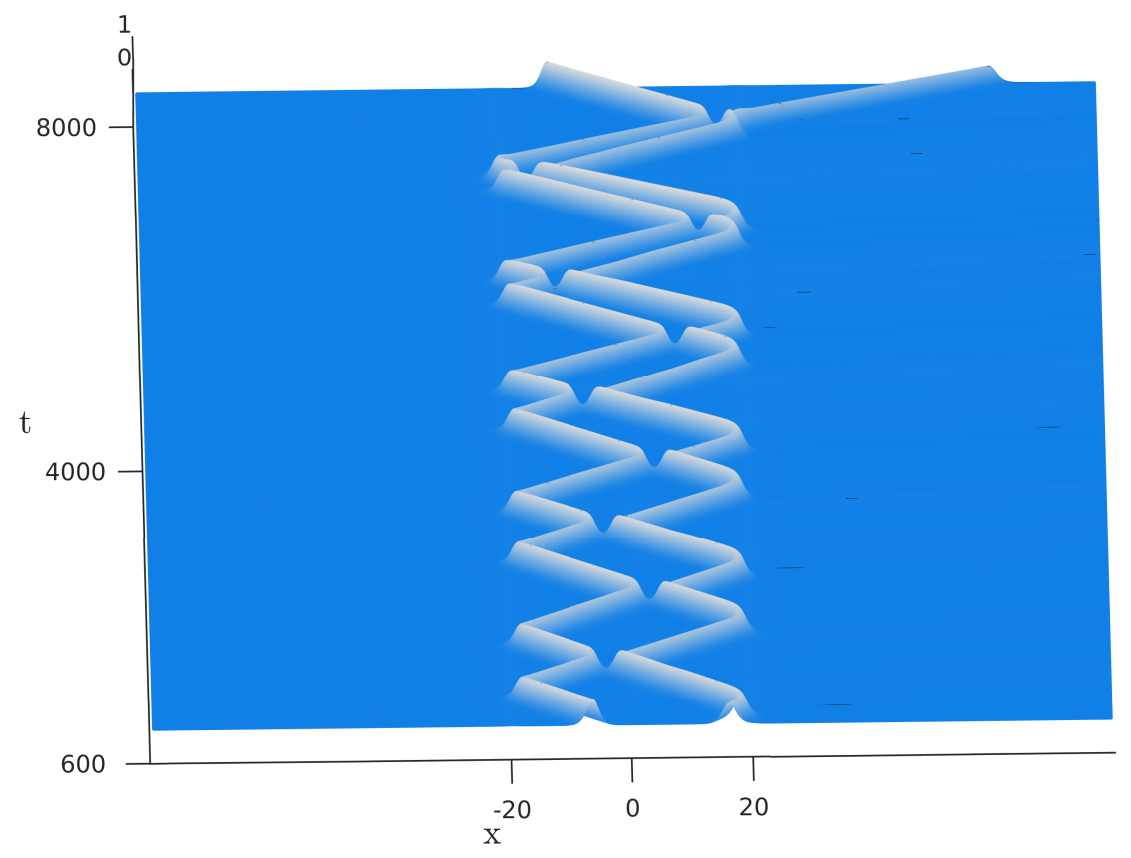

Figure 2: Continuation of figure 1 (top). Parameters $S_{1}=S_{2}=0.6, \phi=16$ and $\psi=12$.

only one crest exists. The interaction is characterized by an absorption of the smaller wave and its reemission later, along with a phase lag in the trajectories of the crest, see figure 3 (bottom).

Lastly, we show a collision that presents features similar to the cases (A) and (B) simultaneously, see figure 4. The smaller wave is first swallowed, then expelled by the larger one. This dynamic is very similar to the description given previously in case (C). However, during the collision there is a central region consisting of two crests. This behaviour is described in great details in a serie of snapshots depicted in figure 5 .

For the KdV equation the transition between two categories is determined by the ratio of the amplitudes of the two separated solitary waves given initially. However, for the fKdV is not possible to estimate a similar condition regarding the ratio of the amplitudes as shown in table 1 . Nevertheless the fKdV equations still holds the geometric features of the Lax categorization. 

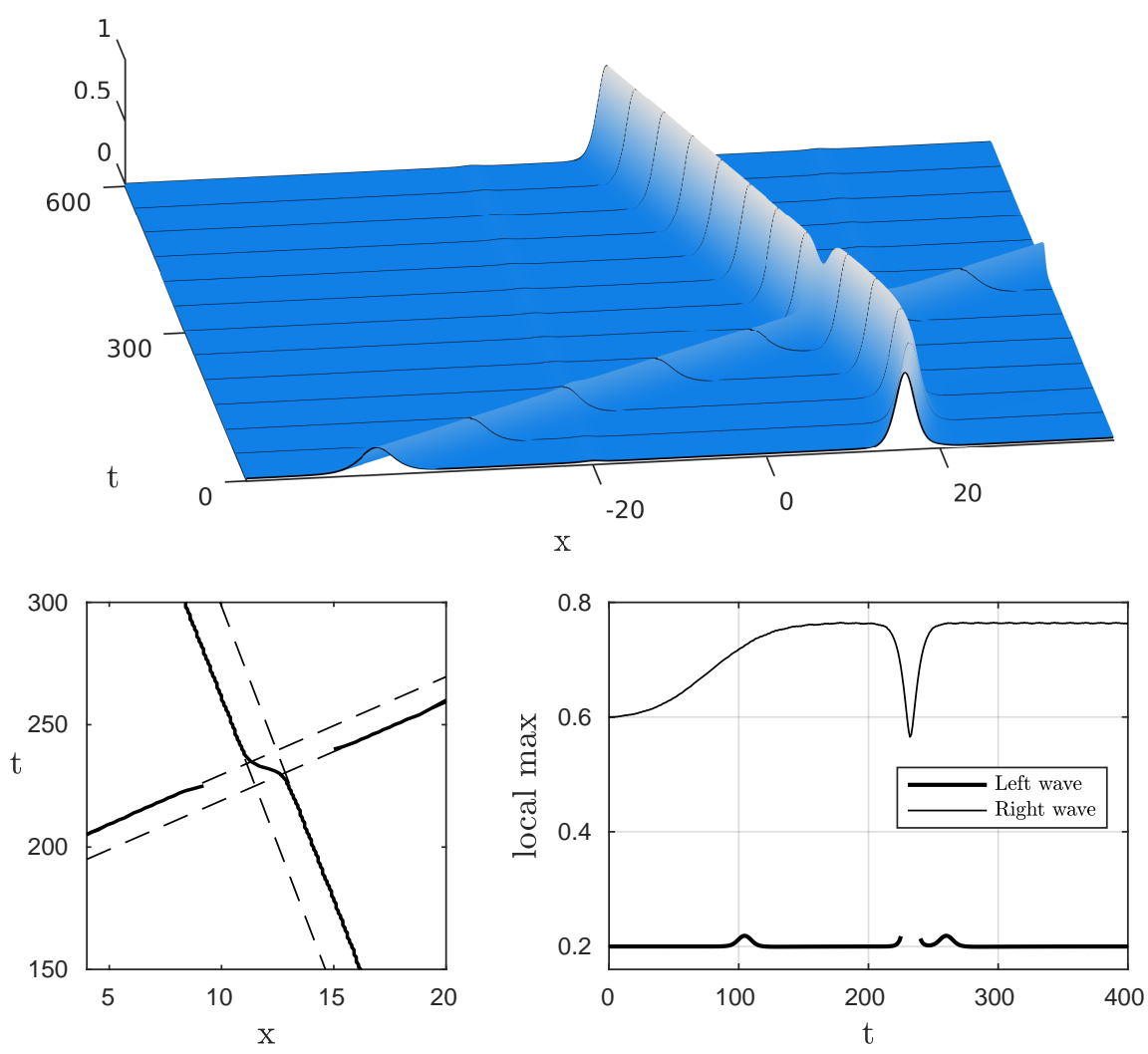

Figure 3: Top: Collision of two well-separate solitary waves - category (C). Bottom (left): Crest trajectory. Bottom (right): The local maxima of the solution as a function of time. Parameters $S_{1}=0.6, S_{2}=0.2, \phi=16$ and $\psi=45$.
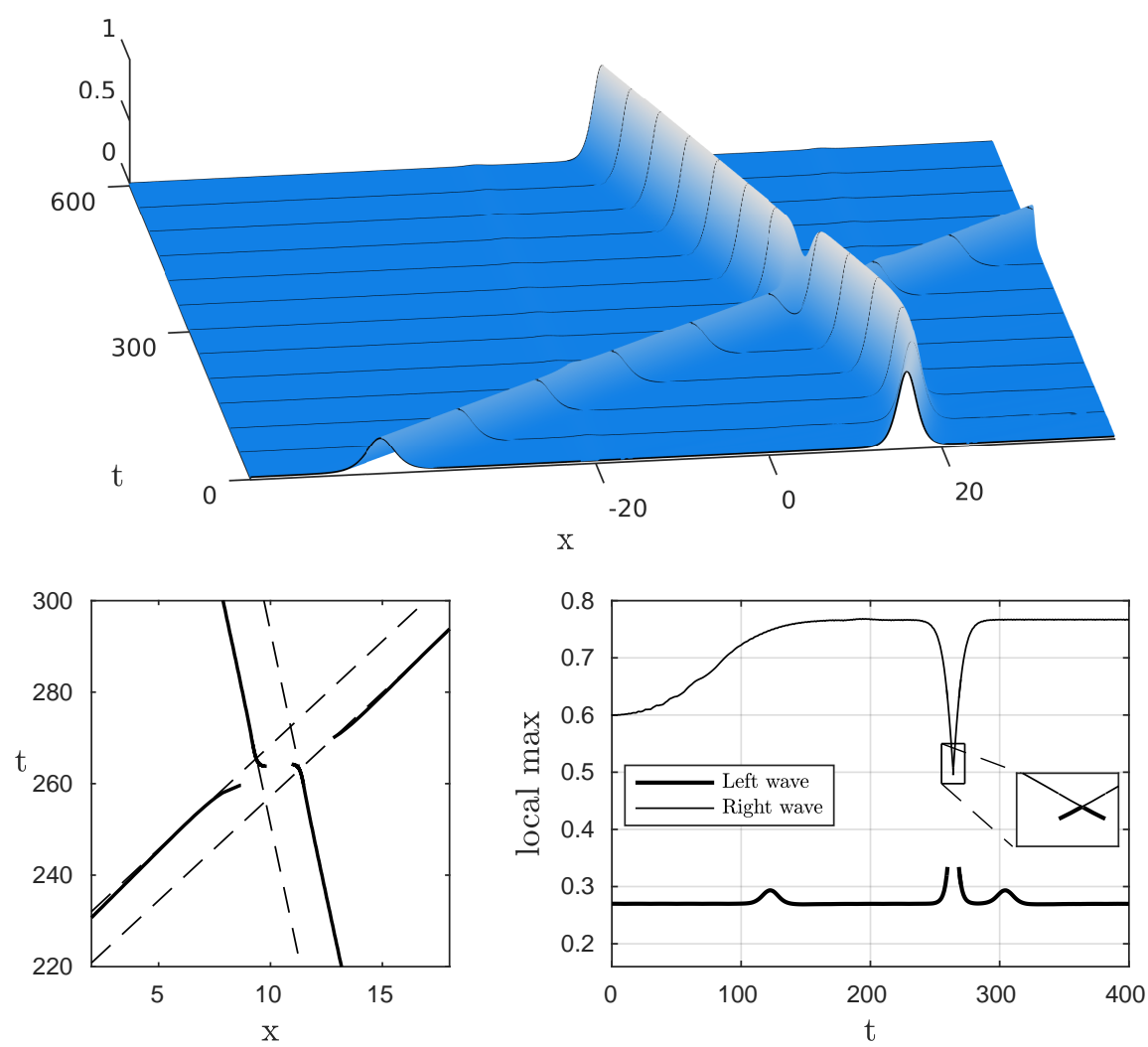

Figure 4: Top: Collision of two well-separate solitary waves - category (B). Bottom (left): Crest trajectory. Bottom (right): The local maxima of the solution as a function of time. Parameters $S_{1}=0.6, S_{2}=0.27, \phi=16$ and $\psi=45$.

\section{Conclusion}

In this paper we have investigated solitary wave collisions for the fKdV equation. Through a pseudospectral numerical method, we showed that the geometric Lax characterisation for the KdV two-soliton interaction still holds for the $\mathrm{fKdV}$, i.e. solitary wave interactions maintain two well separated crests in regime $(\mathbf{A})$, the larger solitary wave absorbs the smaller one and the number of local maxima varies according to the law $2 \rightarrow 1 \rightarrow 2 \rightarrow 1 \rightarrow 2$ in regime (B) or the number of local maxima changes as $2 \rightarrow 1 \rightarrow 2$, case (C). Although there are a number of theoretical and 

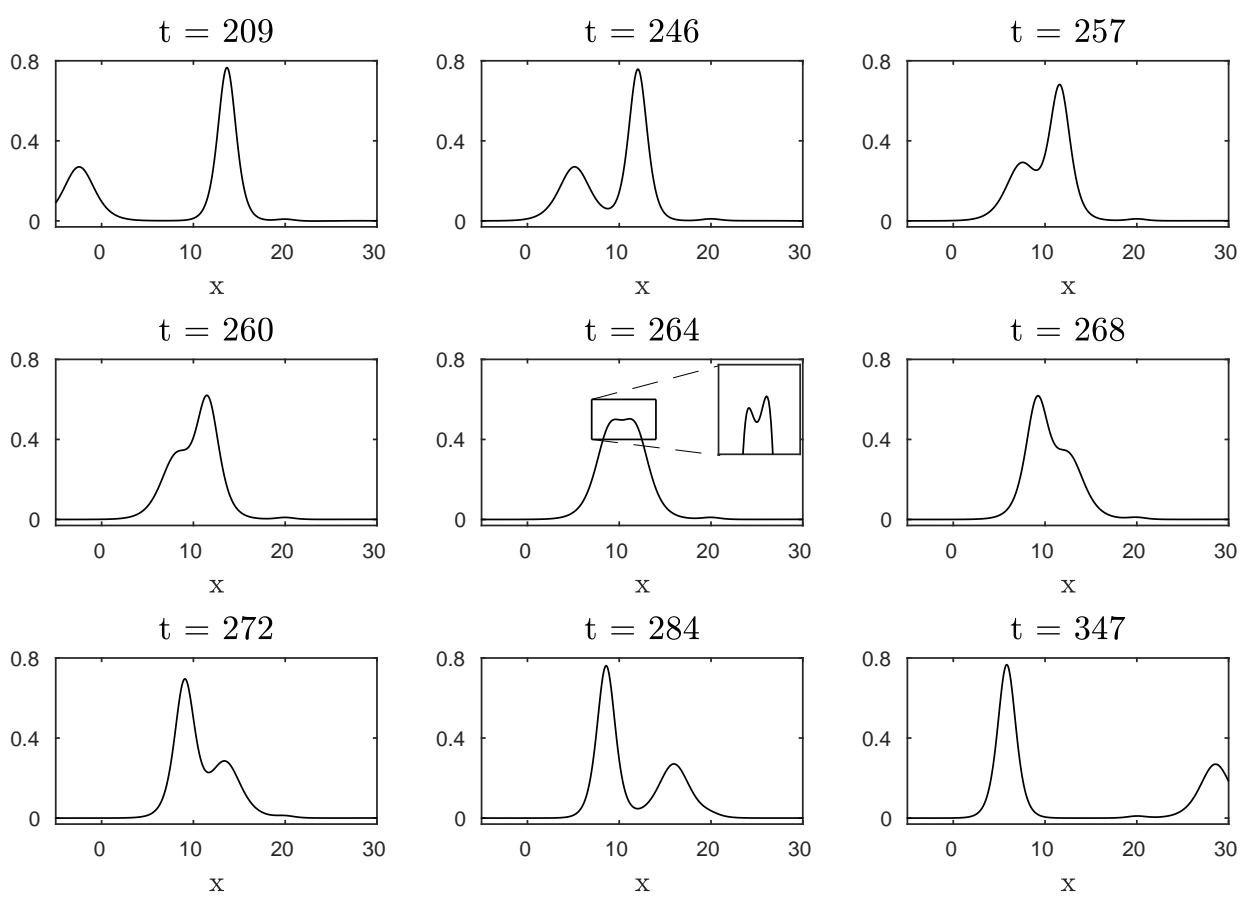

Figure 5: Snapshots of the interaction of the two well-separate solitary waves of figure 4 during the collision - category (B).

\begin{tabular}{c|c|c|c}
\hline \hline$S_{1}$ & $S_{2}$ & $\max \left\{S_{1}, S_{2}\right\} / \min \left\{S_{1}, S_{2}\right\}$ & category \\
\hline 0.60 & 0.30 & 2.00 & $\mathbf{A}$ \\
\hline 0.60 & 0.29 & 2.06 & $\mathbf{A}$ \\
\hline 0.60 & 0.28 & 2.14 & $\mathbf{B}$ \\
\hline 0.60 & 0.27 & 2.22 & $\mathbf{B}$ \\
\hline 0.60 & 0.26 & 2.30 & $\mathbf{B}$ \\
\hline 0.60 & 0.25 & 2.40 & $\mathbf{B}$ \\
\hline 0.60 & 0.24 & 2.50 & $\mathbf{C}$ \\
\hline 0.60 & 0.20 & 3.00 & $\mathbf{C}$ \\
\hline 0.55 & 0.26 & 2.06 & $\mathbf{B}$ \\
\hline 0.55 & 0.27 & 2.00 & $\mathbf{B}$ \\
\hline 0.55 & 0.37 & 1.50 & $\mathbf{A}$ \\
\hline
\end{tabular}

Table 1: Classification of the collision for different values of $S_{1}$ and $S_{2}$.

numerical works on collisions for the KdV equation, as far as we know there are no articles focused on collision details for the fKdV equation.

\section{Acknowledgements}

The authors are grateful to IMPA-National Institute of Pure and Applied Mathematics for the research support provided during the Summer Program of 2020 to Prof. Paul Mileswki (University of Bath) for his constructive comments and suggestions which improved the manuscript. M.F. is grateful to Federal University of Paraná for the visit to the Department of Mathematics. R.R.-Jr is grateful to University of Bath for the extended visit to the Department of Mathematical Sciences.

\section{References}

BAines, P. 1995 Topographic effects in stratified flows. Cambridge University Press.

Craig, W., Guynne, P., Hammack, J., Henderson, D. \& Sulem, C. 2006 Solitary water wave interactions. Phys. Fluids. 18.

Flamarion, M. V., Milewski, P. A. \& Nachbin A. 2019 Rotational waves generated by currenttopography interaction. Stud Appl Math, 142, 433-464.

Grimshaw, R. \& Maleewong, M. 2013 Stability of steady gravity waves generated by a moving localized pressure disturbance in water of finite depth. Phys Fluids, 25.

Joseph. A. 2016 Investigating Seaflaws in the Oceans New York: Elsevier. 
Johnson, R. S. 2012 Models for the formation of a critical layer in water wave propagation. Phil. Trans. R. Soc. A 370, 1638-1660.

LAX, P. D. 1968 Integrals of Nonlinear Equations of Evolution and Solitary Waves. Commun. Pur. Appl. Math. 21, 467-490.

Milewski, P. A. 2004 The Forced Korteweg-de Vries Equation as a Model for Waves Generated by Topography. CUBO A mathematical Journal 6 (4), 33-51.

Mirie, R. M. \& Su, C. H. 1982 Collisions between two solitary waves. Part 2. A numerical study. J. Fluid Mech. 115, 475-492.

Pratt, LJ. 1984 On nonlinear flow with multiple obstructions. J. Atmos. Sci. 41 , 1214-1225.

Trefethen, L. N. 2001 Spectral Methods in MATLAB. Philadelphia: SIAM.

Weidman, P. D. \& Maxworthy, T. 1978 Experiments on strong interaction between solitary waves $J$ Fluid Mech 85, 417-431.

Wu, T. Y. 1987 Generation of upstream advancing solitons by moving disturbances J Fluid Mech $184,75-99$.

Zabusky, M. \& KRUskal, N. 1965 Interaction of solitons in a collisionless plasma and the recurrence of initial states Phys. Rev. Lett., 15, 240-243. 